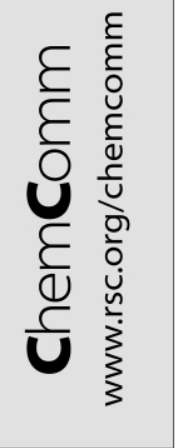

\title{
A new method for the synthesis of hydrophobized, catalytically active Pt nanoparticles
}

\author{
Saikat Mandal, ${ }^{a}$ PR. Selvakannan, ${ }^{a}$ Debdut Roy, ${ }^{b}$ Raghunath V. Chaudhari ${ }^{b}$ and Murali Sastry*a \\ a Materials Chemistry Division, National Chemical Laboratory, Pune 411 008, India. \\ E-mail: sastry@ems.ncl.res.in \\ ${ }^{b}$ Homogeneous Catalysis Divisions, National Chemical Laboratory, Pune 411 008, India
}

Received (in Cambridge, UK) 18th September 2002, Accepted 28th October 2002

First published as an Advance Article on the web 13th November 2002

A single step method for the synthesis of catalytically active, hydrophobic $\mathrm{Pt}$ nanoparticles by the spontaneous reduction of aqueous $\mathrm{PtCl}_{6}{ }^{2-}$ ions by hexadecylaniline molecules at a liquid-liquid interface is described.

Development of protocols for the synthesis of metal nanoparticles of variable size and shape constitutes an area of research of considerable topical interest. Exciting application of metal nanoparticles in electronics, ${ }^{1}$ magnetic memories, ${ }^{2}$ biolabelling/biosensors ${ }^{3}$ and catalysis ${ }^{4}$ has to a large extent motivated research in synthesis methodologies. Application of metal nanoparticles in catalysis has predictably centred around $\mathrm{Pt}$ and $\mathrm{Pd}$ nanoparticles.5,6 As far as $\mathrm{Pt}$ nanoparticles are concerned, a number of processes have been developed for their synthesis in an aqueous environment, ${ }^{5-7}$ in non-polar organic solutions 8,9 and by phase transfer of aqueous nanoparticles to organic solutions. ${ }^{10,11}$ One of the more popular methods for the synthesis of hydrophobized Pt nanoparticles in an organic medium involves the Schiffrin method wherein aqueous $\mathrm{PtCl}_{6}{ }^{2-}$ ions are transferred to non-polar organic solvents by phase-transfer molecules such as tetraalkylammonium salts, reduced by borohydride treatment and finally capped with stabilizing alkyl isocyanide molecules. ${ }^{8}$ Very recently, we have shown that the Brust process for synthesis of organically dispersible gold nanoparticles may be simplified to a one-step method by using the multifunctional molecule 4-hexadecylaniline (HDA) in the organic phase, this molecule playing the simultaneous role of a phase-transfer molecule, reducing and nanoparticle capping agent. ${ }^{12}$ In this communication, we demonstrate that our process works equally well with aqueous $\mathrm{PtCl}_{6}{ }^{2-}$ ions resulting in the formation of extremely stable hydrophobized Pt nanoparticles indicating that HDA molecules are fairly strong reducing agents. Furthermore, the HDAcapped Pt nanoparticles show excellent catalytic activity in the hydrogenation of styrene to ethylbenzene. This is an important result given that capping of $\mathrm{Pt}$ nanoparticles by alkanethiols is known to dramatically reduce their catalytic activity. ${ }^{5}$

In a typical experiment, $50 \mathrm{ml}$ of a $10^{-3} \mathrm{M}$ aqueous solution of $\mathrm{H}_{2} \mathrm{PtCl}_{6}$ was taken in a beaker along with $50 \mathrm{ml}$ solution of $10^{-3} \mathrm{M}$ HDA in chloroform. The biphasic mixture was stirred vigorously on a magnetic stirrer for $56 \mathrm{~h}$ in the dark following which the appearance of brownish colour in the organic phase could clearly be seen. The brown colour in the chloroform phase is a clear indication of the formation of well-dispersed $\mathrm{Pt}$ nanoparticles. ${ }^{8,9}$ After completion of the reaction, the chloroform solution was rotovapped yielding a blackish powder of the Pt nanoparticles stabilized by HDA. This powder was washed repeatedly with methanol to remove uncoordinated HDA molecules and could be readily redispersed in solvents such as benzene, toluene, hexane etc. indicating that HDA-functionalization protects the particles against aggregation during drying. For catalytic activity measurements, a known amount of the purified Pt nanoparticle powder was dispersed in cyclohexane.

Fig. 1A shows the UV-vis spectrum recorded from the HDAstabilized Pt nanoparticles redispersed in chloroform. $\dagger$ A sharp resonance at $c a .258 \mathrm{~nm}$ is observed from the nanoparticle solution. This absorption band is due to excitation of surface plasmon vibrations in the Pt nanoparticles. The position of this absorption band and the nature of the absorption tail is similar to that reported by others for solutions of Pt nanoparticles. ${ }^{9}$ The inset of Fig. 1A shows the X-ray diffraction (XRD) pattern recorded from a drop-coated film of the hydrophobized $\mathrm{Pt}$ nanoparticles. The positions of the Bragg reflections (indexed in the figure) correspond very well with those reported in the literature for face centred cubic (fcc) Pt. ${ }^{13}$ Fig. 1B shows a representative transmission electron microscopy (TEM) image recorded from the HDA-capped Pt nanoparticles drop-coated onto a TEM grid. A large number of Pt nanoparticles of uniform size are observed in the TEM image. A magnified view of the region defined by the box is also shown in Fig. 1B to get a clearer idea of the average particle size and degree of monodispersity. From an analysis of the Pt nanoparticles in this and other images, an average particle size of $15.5 \pm 0.7 \mathrm{~nm}$ was determined. Thus, the process of spontaneous reducion of $\mathrm{PtCl}_{6}{ }^{2-}$ ions by HDA leads to very uniform $\mathrm{Pt}$ nanoparticles, possibly due to capping of the nanoparticles, which is also carried out by HDA. Another interesting feature of the TEM image is the very regular separation between the Pt nanoparticles. This is clearly due to the HDA molecules capping the nanoparticles and preventing their aggregation after solvent evaporation. An average inter-particle distance of $c a .4 .5 \mathrm{~nm}$ was determined and is smaller than the separation expected from the size of the HDA molecules $(\sim 2 \times 2.9 \mathrm{~nm})$. This indicates interdigitation of the hydrocarbon chains from neighbouring nanoparticles.

In order to understand better the nature of interaction of the HDA molecules with the Pt nanoparticle surface, thermogravimetric analysis (TGA) of carefully weighed amount of the purified powder was carried out and the data obtained is shown in Fig. 2A. A weight loss of $\mathrm{ca}$. $30 \%$ is observed to occur at 270 ${ }^{\circ} \mathrm{C}$ and is attributed to desorption of surface-bound HDA molecules. The magnitiude of weight loss and temperature is almost identical to that observed in our earlier study on HDA complexed with gold nanoparticles ${ }^{12}$ indicating similar binding in both cases. The almost $100 \%$ weight loss above $450{ }^{\circ} \mathrm{C}$ for $\mathrm{Pt}$

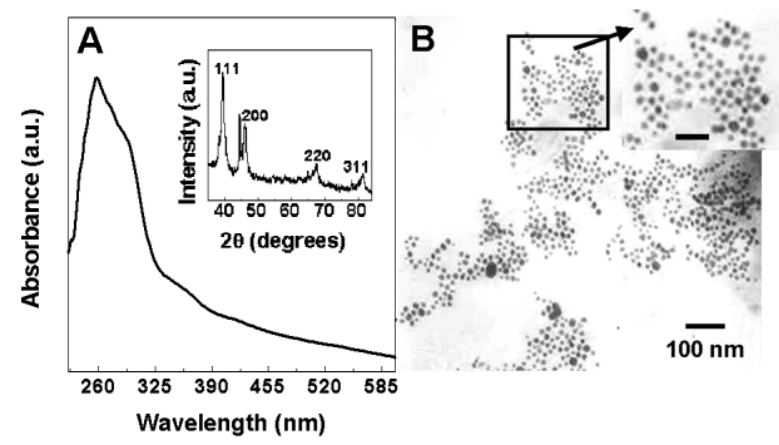

Fig. 1 (A) UV-vis spectrum recorded from HDA-capped Pt nanoparticles redispersed in chloroform (see text for details). The inset shows the XRD pattern obtained from a drop-coated HDA-capped Pt nanoparticle film. (B) TEM image of a film of HDA-capped Pt nanoparticles drop-coated from chloroform onto a TEM grid. A magnified view of the region enclosed by a box is shown in the top right corner (scale bar $=50 \mathrm{~nm}$ ). 

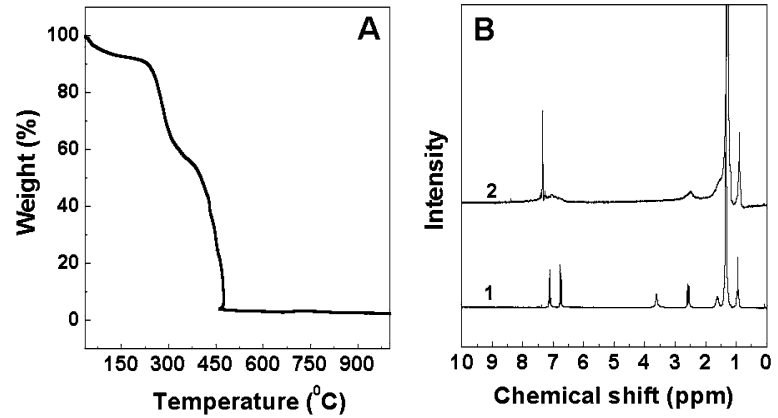

Fig. 2 (A) TGA data recorded from the HDA-capped Pt nanoparticle powder. (B) ${ }^{1} \mathrm{H}$ NMR spectra of pure HDA (curve 1) and HDA reduced $\mathrm{Pt}$ nanoparticles (curve 2).

nanoparticles is also a feature common to both systems ${ }^{12}$ and indicates complete desorption of the nanoparticles at this relatively low temperature.

The changes occuring in the HDA molecules consequent to reduction of the chloroplatinate ions may be conveniently studied by NMR spectroscopy. Fig. 2B shows the results of proton NMR measurements carried out on pure HDA (curve 1) and HDA-reduced platinum nanoparticles (curve 2) redispersed in $\mathrm{CDCl}_{3}$ after purification in order to understand better changes occurring in HDA during the reduction of aqueous hexachloroplatinate ions. The chemical shifts at 7.1 and $6.75 \mathrm{ppm}$ in curve 1 (pure HDA) are assigned to the two sets of aromatic protons while the chemical shifts at 3.64, 2.56, 1.32 and 0.91 ppm correspond to the $\mathrm{NH}_{2}$ protons, methylene protons attached to the benzene ring, other methylene protons in the hydrocarbon chain and methyl protons in the pure HDA molecule respectively. In curve 2 (HDA-reduced platinum nanoparticles) there is a complete disappearance of the peaks at $7.1,6.75$ and $3.64 \mathrm{ppm}$ and a broadening of the peak at 2.56 ppm. This indicates that the aniline group in HDA is in close contact with the metal surface with the hydrocarbon chain pointing outwards. We would like to point out that this behaviour is different from alkylamines bound to gold nanoparticles where the peaks for amine-capped Au nanocrystals are all broadened relative to those of the neat amines and a peak at $2.70 \mathrm{ppm}$, typical of the $\mathrm{NCH}_{2}$ protons in the neat amines, is not clearly observed in the amine-capped Au nanocrystal spectra. ${ }^{14}$

To the best of our knowledge, there are no reports on the catalytic activity of hydrophobized Pt nanoparticles in organic media and we were therefore curious to test whether HDAstabilized $\mathrm{Pt}$ nanoparticles retained any catalytic activity. Catalytic activity of the platinum nanoparticles for a hydrogenation reaction involving the conversion of styrene to ethylbenzene (Scheme 1) was studied in a $50 \mathrm{ml}$ reactor (Hastelloy-C, supplied by Parr Instrument Company, USA).

Analysis of the initial and final samples were done using gas chromatography (HP 6890) using an HP-FFAP capillary

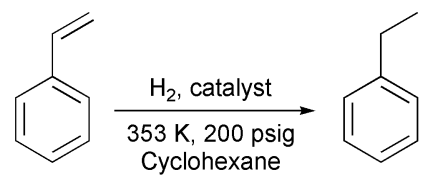

Scheme 1 column (poly(ethylene glycol)-TPA as the stationary phase, 25 $\mathrm{m} \times 200 \mu \mathrm{m} \times 0.3 \mu \mathrm{m})$ having conditions: $403 \mathrm{~K}$ for $2 \mathrm{~min} @$ $20 \mathrm{~K} \mathrm{~min}^{-1}$ up to $423 \mathrm{~K}$-hold 2 min-@ $@ 20 \mathrm{~K} \mathrm{~min}^{-1}$ up to 463 $\mathrm{K}$-hold $1 \mathrm{~min}$; inlet temp: $473 \mathrm{~K}$, inlet pressure: $25 \mathrm{psig}$; detector temp: $523 \mathrm{~K}$. The GC analysis showed complete conversion of styrene with $99.81 \%$ selectivity to ethylbenzene with a TOF of $655 \mathrm{~h}^{-1}$. The HDA-capped Pt nanoparticle catalyst also worked excellently in the hydrogenation of a nonaromatic substrate such as cyclohexene giving complete conversion of cyclohexene to cyclohexane with $100 \%$ selectivity $\left(\mathrm{TOF}=2112 \mathrm{~h}^{-1}\right)$.

The use of a multifunctional molecule that accomplishes reduction of aqueous $\mathrm{PtCl}_{6}{ }^{2-}$ ions, caps the $\mathrm{Pt}$ nanoparticles thus formed and renders them dispersible in an organic solvent has been described. The $\mathrm{Pt}$ nanoparticles capped by the multifunctional molecule, hexadecylaniline, are highly active and catalyze the hydrogenation of styrene to ethylbenzene and cyclohexene to cyclohexane with almost $100 \%$ selectivity.

S. M., PR. S. and D. R. thank the University Grants Commission and the Council of Scientific and Industrial Research (CSIR), respectively, Govt. of India, for financial support. The authors thank Ms Renu Pasricha and Ms S.D. Adyanthaya for assistance with the TEM and TGA measurements, respectively.

\section{Notes and references}

$\dagger \mathrm{UV}$-vis spectra of the Pt nanoparticle solution in chloroform were measured on a Hewlett-Packard HP 8452 diode array spectrophotometer at a resolution of $2 \mathrm{~nm}$. TEM measurements on the HDA-capped $\mathrm{Pt}$ nanoparticle films cast onto carbon-coated TEM grids were carried out on a JEOL model 1200EX instrument at an accelerating voltage of $120 \mathrm{kV}$. TGA profiles of carefully weighed quantities of purified powders of HDAcapped platinum nanoparticles were recorded on a Seiko Instruments model TG/DTA 32 instrument at a heating rate of $10{ }^{\circ} \mathrm{C} \mathrm{min}^{-1}$. XRD measurements on drop-coated HDA-capped Pt nanoparticle films on a glass substrate were carried out on a Phillips PW 1830 instrument in the transmission at a voltage of $40 \mathrm{kV}$ and current of $30 \mathrm{~mA}$ with $\mathrm{Cu} \mathrm{K \alpha}$ radiation. The proton $\left({ }^{1} \mathrm{H} \mathrm{NMR}\right) \mathrm{NMR}$ spectra were recorded on a Bruker AC $200 \mathrm{MHz}$ instrument and scanned in the range $0-15 \mathrm{ppm}$.

1 G. Schon and U. Simon, Colloid Polym. Sci., 1995, 273, 202.

2 J. M. Thomas, Pure Appl. Chem., 1988, 60, 1517.

3 S. J. Park, A. A. Lazarides, C. A. Mirkin, P. W. Brazis, C. R. Kannewurf and C. R. Letsinger, Angew. Chem., Int. Ed., 2000, 39, 3845.

4 H. Hirai, H. Wakabayashi and M. Komiyama, Chem. Lett., 1983, 1047.

5 R. M. Crooks, M. Zhao, L. Sun, V. Chechik and L. K. Yeung, Acc. Chem. Res., 2001, 34, 181.

6 J. Alvarez, J. Liu and A. E. Kaifer, Chem. Commun., 2000, 1151.

7 Y. Mizukoshi, R. Oshima, Y. Maeda and Y. Nagata, Langmuir, 1999, 15, 2733.

8 S. L. Horswell, C. J. Kiely, I. A. O'Neil and D. J. Schiffrin, J. Am. Chem. Soc., 1999, 121, 5573.

9 C. Yee, M. Scotti, A. Ulman, H. White, M. Rafailovich and J. Sokolov, Langmuir, 1999, 15, 4313.

10 K. V. Sarathy, G. Raina, R. T. Yadav, G. U. Kulkarni and C. N. R. Rao, J. Phys. Chem. B., 1997, 101, 9876.

11 S-Y. Zhao, S-H. Chen, S-Y. Wang, D-G. Li and H-Y. Ma, Langmuir, $2002, \mathbf{1 8}, 3315$.

12 PR. Selvakannan, S. Mandal, R. Pasricha, S. D. Adyanthaya and M. Sastry, Chem. Commun., 2002, 1334.

13 H. Perez, J.-P. Pradeau, P.-A. Albouy and J. Perez-Omil, Chem. Mater., 1999, 11, 3460

14 D. V. Leff, L. Brandt and J. R. Heath, Langmuir, 1996, 12, 4723. 